hep-ex/0308057

\title{
Measurement of the Branching Fraction and Polarization for the Decay $B^{-} \rightarrow D^{* 0} K^{*-}$
}

B. Aubert,${ }^{1}$ R. Barate,${ }^{1}$ D. Boutigny, ${ }_{1}^{1}$ J.-M. Gaillard,${ }^{1}$ A. Hicheur, ${ }^{1}$ Y. Karyotakis, ${ }^{1}$ J. P. Lees, ${ }^{1}$ P. Robbe,${ }^{1}$ V. Tisserand,${ }^{1}$ A. Zghiche, ${ }^{1}$ A. Palano, ${ }^{2}$ A. Pompili, ${ }^{2}$ J. C. Chen,${ }^{3}$ N. D. Qi,${ }^{3}$ G. Rong, ${ }^{3}$ P. Wang, ${ }^{3}$ Y. S. Zhu, ${ }^{3}$ G. Eigen, ${ }^{4}$ I. Ofte ${ }^{4}$ B. Stugu ${ }^{4}$ G. S. Abrams,${ }^{5}$ A. W. Borgland,${ }^{5}$ A. B. Breon, ${ }^{5}$ D. N. Brown, ${ }^{5}$ J. Button-Shafer, ${ }^{5}$ R. N. Cahn, ${ }^{5}$ E. Charles,${ }^{5}$ C. T. Day, ${ }^{5}$ M. S. Gill, ${ }^{5}$ A. V. Gritsan, ${ }^{5}$ Y. Groysman,${ }^{5}$ R. G. Jacobsen, ${ }^{5}$ R. W. Kadel,${ }^{5}$ J. Kadyk, ${ }^{5}$ L. T. Kerth, ${ }^{5}$ Yu. G. Kolomensky ${ }^{5}$ J. F. Kral,${ }^{5}$ G. Kukartsev ${ }^{5}$ C. LeClerc, ${ }^{5}$ M. E. Levi, ${ }^{5}$ G. Lynch, ${ }^{5}$ L. M. Mir, ${ }^{5}$ P. J. Oddone,${ }^{5}$ T. J. Orimoto,${ }^{5}$ M. Pripstein, ${ }^{5}$ N. A. Roe,${ }^{5}$ A. Romosan, ${ }^{5}$ M. T. Ronan, ${ }^{5}$ V. G. Shelkov ${ }^{5}$

A. V. Telnov ${ }^{5}$ W. A. Wenzel,${ }^{5}$ K. Ford,${ }^{6}$ T. J. Harrison,${ }^{6}$ C. M. Hawkes,${ }^{6}$ D. J. Knowles, ${ }^{6}$ S. E. Morgan,${ }^{6}$

R. C. Penny, ${ }^{6}$ A. T. Watson,${ }^{6}$ N. K. Watson, ${ }^{6}$ K. Goetzen, ${ }^{7}$ T. Held,${ }^{7}$ H. Koch, ${ }^{7}$ B. Lewandowski,${ }^{7}$ M. Pelizaeus, ${ }^{7}$

K. Peters,${ }^{7}$ H. Schmuecker, ${ }^{7}$ M. Steinke, ${ }^{7}$ N. R. Barlow, ${ }^{8}$ J. T. Boyd,${ }^{8}$ N. Chevalier, ${ }^{8}$ W. N. Cottingham, ${ }^{8}$

M. P. Kelly ${ }^{8}$ T. E. Latham,${ }^{8}$ C. Mackay,${ }^{8}$ F. F. Wilson, ${ }^{8}$ K. Abe,${ }^{9}$ T. Cuhadar-Donszelmann, ${ }^{9}$ C. Hearty,${ }^{9}$

T. S. Mattison, ${ }^{9}$ J. A. McKenna, ${ }^{9}$ D. Thiessen,${ }^{9}$ P. Kyberd,${ }^{10}$ A. K. McKemey, ${ }^{10}$ V. E. Blinov, ${ }^{11}$ A. D. Bukin, ${ }^{11}$

V. B. Golubev, ${ }^{11}$ V. N. Ivanchenko, ${ }^{11}$ E. A. Kravchenko, ${ }^{11}$ A. P. Onuchin, ${ }^{11}$ S. I. Serednyakov, ${ }^{11}$ Yu. I. Skovpen, ${ }^{11}$

E. P. Solodov, ${ }^{11}$ A. N. Yushkov, ${ }^{11}$ D. Best,,${ }^{12}$ M. Bruinsma, ${ }^{12}$ M. Chao,${ }^{12}$ D. Kirkby, ${ }^{12}$ A. J. Lankford,${ }^{12}$

M. Mandelkern, ${ }^{12}$ R. K. Mommsen, ${ }^{12}$ W. Roethel,${ }^{12}$ D. P. Stoker, ${ }^{12}$ C. Buchanan, ${ }^{13}$ B. L. Hartfiel,${ }^{13}$ B. C. Shen, ${ }^{14}$

D. del Re, ${ }^{15}$ H. K. Hadavand, ${ }^{15}$ E. J. Hill,,${ }^{15}$ D. B. MacFarlane, ${ }^{15}$ H. P. Paar, ${ }^{15}$ Sh. Rahatlou, ${ }^{15}$ V. Sharma, ${ }^{15}$

J. W. Berryhill, ${ }^{16}$ C. Campagnari, ${ }^{16}$ B. Dahmes,${ }^{16}$ N. Kuznetsova, ${ }^{16}$ S. L. Levy, ${ }^{16}$ O. Long, ${ }^{16}$ A. Lu, ${ }^{16}$

M. A. Mazur, ${ }^{16}$ J. D. Richman, ${ }^{16}$ W. Verkerke,${ }^{16}$ T. W. Beck,,${ }^{17}$ J. Beringer,${ }^{17}$ A. M. Eisner, ${ }^{17}$ C. A. Heusch, ${ }^{17}$

W. S. Lockman, ${ }^{17}$ T. Schalk, ${ }^{17}$ R. E. Schmitz, ${ }^{17}$ B. A. Schumm, ${ }^{17}$ A. Seiden, ${ }^{17}$ M. Turri, ${ }^{17}$ W. Walkowiak, ${ }^{17}$

D. C. Williams, ${ }^{17}$ M. G. Wilson, ${ }^{17}$ J. Albert,${ }^{18}$ E. Chen, ${ }^{18}$ G. P. Dubois-Felsmann, ${ }^{18}$ A. Dvoretskii, ${ }^{18}$ D. G. Hitlin,${ }^{18}$

I. Narsky, ${ }^{18}$ F. C. Porter,${ }^{18}$ A. Ryd,${ }^{18}$ A. Samuel, ${ }^{18}$ S. Yang, ${ }^{18}$ S. Jayatilleke,${ }^{19}$ G. Mancinelli, ${ }^{19}$ B. T. Meadows,${ }^{19}$

M. D. Sokoloff, ${ }^{19}$ T. Abe,${ }^{20}$ F. Blanc,${ }^{20}$ P. Bloom,${ }^{20}$ S. Chen, ${ }^{20}$ P. J. Clark,${ }^{20}$ W. T. Ford,${ }^{20}$ U. Nauenberg, ${ }^{20}$

A. Olivas,${ }^{20}$ P. Rankin, ${ }^{20}$ J. Roy, ${ }^{20}$ J. G. Smith, ${ }^{20}$ W. C. van Hoek ${ }^{20}$ L. Zhang, ${ }^{20}$ J. L. Harton, ${ }^{21}$ T. Hu, ${ }^{21}$

A. Soffer, ${ }^{21}$ W. H. Toki,${ }^{21}$ R. J. Wilson, ${ }^{21}$ J. Zhang, ${ }^{21}$ D. Altenburg, ${ }^{22}$ T. Brandt, ${ }^{22}$ J. Brose,${ }^{22}$ T. Colberg, ${ }^{22}$

M. Dickopp ${ }^{22}$ R. S. Dubitzky ${ }^{22}$ A. Hauke,${ }^{22}$ H. M. Lacker, ${ }^{22}$ E. Maly ${ }^{22}$ R. Müller-Pfefferkorn, ${ }^{22}$ R. Nogowski, ${ }^{22}$

S. Otto, ${ }^{22}$ J. Schubert, ${ }^{22}$ K. R. Schubert, ${ }^{22}$ R. Schwierz,${ }^{22}$ B. Spaan, ${ }^{22}$ L. Wilden, ${ }^{22}$ D. Bernard,${ }^{23}$

G. R. Bonneaud, ${ }^{23}$ F. Brochard, ${ }^{23}$ J. Cohen-Tanugi, ${ }^{23}$ P. Grenier, ${ }^{23}$ Ch. Thiebaux, ${ }^{23}$ G. Vasileiadis, ${ }^{23}$ M. Verderi, ${ }^{23}$ A. Khan, ${ }^{24}$ D. Lavin, ${ }^{24}$ F. Muheim, ${ }^{24}$ S. Playfer, ${ }^{24}$ J. E. Swain,${ }^{24}$ M. Andreotti, ${ }^{25}$ V. Azzolini, ${ }^{25}$ D. Bettoni, ${ }^{25}$ C. Bozzi,${ }^{25}$ R. Calabrese,${ }^{25}$ G. Cibinetto,${ }^{25}$ E. Luppi,${ }^{25}$ M. Negrini,${ }^{25}$ L. Piemontese,${ }^{25}$ A. Sarti, ${ }^{25}$ E. Treadwell, ${ }^{26}$ F. Anulli, ${ }^{27}, *$ R. Baldini-Ferroli, ${ }^{27}$ M. Biasini, ${ }^{27}, *$ A. Calcaterra,,${ }^{27}$ R. de Sangro, ${ }^{27}$ D. Falciai, ${ }^{27}$ G. Finocchiaro, ${ }^{27}$

P. Patteri,${ }^{27}$ I. M. Peruzzi, ${ }^{27, *}$ M. Piccolo, ${ }^{27}$ M. Pioppi,${ }^{27, *}$ A. Zallo, ${ }^{27}$ A. Buzzo, ${ }^{28}$ R. Capra, ${ }^{28}$ R. Contri ${ }^{28}$

G. Crosetti, ${ }^{28}$ M. Lo Vetere,${ }^{28}$ M. Macri, ${ }^{28}$ M. R. Monge,${ }^{28}$ S. Passaggio, ${ }^{28}$ C. Patrignani, ${ }^{28}$ E. Robutti, ${ }^{28}$

A. Santroni, ${ }^{28}$ S. Tosi, ${ }^{28}$ S. Bailey, ${ }^{29}$ M. Morii, ${ }^{29}$ E. Won,${ }^{29}$ W. Bhimji, ${ }^{30}$ D. A. Bowerman,${ }^{30}$ P. D. Dauncey, ${ }^{30}$

U. Egede ${ }^{30}$ I. Eschrich, ${ }^{30}$ J. R. Gaillard, ${ }^{30}$ G. W. Morton, ${ }^{30}$ J. A. Nash, ${ }^{30}$ P. Sanders,${ }^{30}$ G. P. Taylor,${ }^{30}$

G. J. Grenier, ${ }^{31}$ S.-J. Lee, ${ }^{31}$ U. Mallik, ${ }^{31}$ J. Cochran, ${ }^{32}$ H. B. Crawley ${ }^{32}$ J. Lamsa ${ }^{32}$ W. T. Meyer, ${ }^{32}$ S. Prell, ${ }^{32}$

E. I. Rosenberg, ${ }^{32}$ J. Yi,${ }^{32}$ M. Davier,${ }^{33}$ G. Grosdidier, ${ }^{33}$ A. Höcker ${ }^{33}$ S. Laplace, ${ }^{33}$ F. Le Diberder, ${ }^{33}$ V. Lepeltier,${ }^{33}$

A. M. Lutz ${ }^{33}$ T. C. Petersen, ${ }^{33}$ S. Plaszczynski, ${ }^{33}$ M. H. Schune, ${ }^{33}$ L. Tantot,${ }^{33}$ G. Wormser ${ }^{33}$ V. Brigljević,${ }^{34}$

C. H. Cheng, ${ }^{34}$ D. J. Lange, ${ }^{34}$ D. M. Wright,${ }^{34}$ A. J. Bevan, ${ }^{35}$ J. P. Coleman, ${ }^{35}$ J. R. Fry ${ }^{35}$ E. Gabathuler, ${ }^{35}$

R. Gamet, ${ }^{35}$ M. Kay ${ }^{35}$ R. J. Parry ${ }^{35}$ D. J. Payne ${ }^{35}$ R. J. Sloane, ${ }^{35}$ C. Touramanis, ${ }^{35}$ J. J. Back, ${ }^{36}$ P. F. Harrison, ${ }^{36}$

H. W. Shorthouse,${ }^{36}$ P. Strother ${ }^{36}$ P. B. Vidal, ${ }^{36}$ C. L. Brown, ${ }^{37}$ G. Cowan, ${ }^{37}$ R. L. Flack, ${ }^{37}$ H. U. Flaecher,${ }^{37}$

S. George, ${ }^{37}$ M. G. Green, ${ }^{37}$ A. Kurup,${ }^{37}$ C. E. Marker, ${ }^{37}$ T. R. McMahon,${ }^{37}$ S. Ricciardi, ${ }^{37}$ F. Salvatore, ${ }^{37}$

G. Vaitsas,${ }^{37}$ M. A. Winter, ${ }^{37}$ D. Brown, ${ }^{38}$ C. L. Davis, ${ }^{38}$ J. Allison, ${ }^{39}$ R. J. Barlow, ${ }^{39}$ A. C. Forti, ${ }^{39}$ P. A. Hart,${ }^{39}$

M. C. Hodgkinson ${ }^{39}$ F. Jackson, ${ }^{39}$ G. D. Lafferty, ${ }^{39}$ A. J. Lyon, ${ }^{39}$ J. H. Weatherall, ${ }^{39}$ J. C. Williams, ${ }^{39}$ A. Farbin, ${ }^{40}$

A. Jawahery, ${ }^{40}$ D. Kovalskyi, ${ }^{40}$ C. K. Lae,${ }^{40}$ V. Lillard, ${ }^{40}$ D. A. Roberts, ${ }^{40}$ G. Blaylock,${ }^{41}$ C. Dallapiccola,${ }^{41}$

K. T. Flood ${ }^{41}$ S. S. Hertzbach, ${ }^{41}$ R. Kofler, ${ }^{41}$ V. B. Koptchev,${ }^{41}$ T. B. Moore ${ }^{41}$ S. Saremi,${ }^{41}$ H. Staengle ${ }^{41}$

S. Willocq,${ }^{41}$ R. Cowan, ${ }^{42}$ G. Sciolla,,${ }^{42}$ F. Taylor, ${ }^{42}$ R. K. Yamamoto, ${ }^{42}$ D. J. J. Mangeol,${ }^{43}$ P. M. Patel ${ }^{43}$

A. Lazzaro, ${ }^{44}$ F. Palombo, ${ }^{44}$ J. M. Bauer, ${ }^{45}$ L. Cremaldi, ${ }^{45}$ V. Eschenburg, ${ }^{45}$ R. Godang, ${ }^{45}$ R. Kroeger, ${ }^{45}$

J. Reidy, ${ }^{45}$ D. A. Sanders ${ }^{45}$ D. J. Summers ${ }^{45}$ H. W. Zhao, ${ }^{45}$ S. Brunet,${ }^{46}$ D. Cote-Ahern, ${ }^{46}$ C. Hast,${ }^{46}$ P. Taras,${ }^{46}$

Work supported in part by the Department of Energy contract DE-AC03-76SF00515.

Stanford Linear Accelerator Center, Stanford University, Stanford, CA 94309 
H. Nicholson, ${ }^{47}$ C. Cartaro,${ }^{48}$ N. Cavallo, ${ }^{48}, \dagger$ G. De Nardo,${ }^{48}$ F. Fabozzi $,{ }^{48}, \dagger$ C. Gatto ${ }^{48}$ L. Lista,${ }^{48}$ P. Paolucci, ${ }^{48}$ D. Piccolo, ${ }^{48}$ C. Sciacca, ${ }^{48}$ M. A. Baak, ${ }^{49}$ G. Raven, ${ }^{49}$ J. M. LoSecco, ${ }^{50}$ T. A. Gabriel, ${ }^{51}$ B. Brau, ${ }^{52}$ K. K. Gan, ${ }^{52}$ K. Honscheid, ${ }^{52}$ D. Hufnagel, ${ }^{52}$ H. Kagan, ${ }^{52}$ R. Kass, ${ }^{52}$ T. Pulliam, ${ }^{52}$ Q. K. Wong, ${ }^{52}$ J. Brau, ${ }^{53}$ R. Frey, ${ }^{53}$ C. T. Potter, ${ }^{53}$ N. B. Sinev, ${ }^{53}$ D. Strom, ${ }^{53}$ E. Torrence, ${ }^{53}$ F. Colecchia, ${ }^{54}$ A. Dorigo, ${ }^{54}$ F. Galeazzi, ${ }^{54}$ M. Margoni, ${ }^{54}$ M. Morandin, ${ }^{54}$ M. Posocco, ${ }^{54}$ M. Rotondo, ${ }^{54}$ F. Simonetto, ${ }^{54}$ R. Stroili, ${ }^{54}$ G. Tiozzo, ${ }^{54}$ C. Voci, ${ }^{54}$ M. Benayoun, ${ }^{55}$ H. Briand, ${ }^{55}$ J. Chauveau, ${ }^{55}$ P. David, ${ }^{55}$ Ch. de la Vaissière, ${ }^{55}$ L. Del Buono, ${ }^{55}$ O. Hamon, ${ }^{55}$ M. J. J. John, ${ }^{55}$ Ph. Leruste, ${ }^{55}$ J. Ocariz, ${ }^{55}$ M. Pivk, ${ }^{55}$ L. Roos, ${ }^{55}$ J. Stark, ${ }^{55}$ S. T'Jampens, ${ }^{55}$ G. Therin, ${ }^{55}$ P. F. Manfredi, ${ }^{56}$ V. Re ${ }^{56}$ P. K. Behera, ${ }^{57}$ L. Gladney, ${ }^{57}$ Q. H. Guo, ${ }^{57}$ J. Panetta, ${ }^{57}$ C. Angelini, ${ }^{58}$ G. Batignani, ${ }^{58}$ S. Bettarini, ${ }^{58}$ M. Bondioli, ${ }^{58}$ F. Bucci, ${ }^{58}$ G. Calderini, ${ }^{58}$ M. Carpinelli, ${ }^{58}$ V. Del Gamba, ${ }^{58}$ F. Forti, ${ }^{58}$ M. A. Giorgi, ${ }^{58}$ A. Lusiani, ${ }^{58}$ G. Marchiori, ${ }^{58}$ F. Martinez-Vidal, ${ }^{58, \ddagger}$ M. Morganti, ${ }^{58}$ N. Neri, ${ }^{58}$ E. Paoloni, ${ }^{58}$ M. Rama, ${ }^{58}$ G. Rizzo, ${ }^{58}$ F. Sandrelli, ${ }^{58}$ J. Walsh, ${ }^{58}$ M. Haire ${ }^{59}$ D. Judd, ${ }^{59}$ K. Paick, ${ }^{59}$ D. E. Wagoner, ${ }^{59}$ N. Danielson, ${ }^{60}$ P. Elmer ${ }^{60}$ C. Lu, ${ }^{60}$ V. Miftakov, ${ }^{60}$ J. Olsen, ${ }^{60}$ A. J. S. Smith, ${ }^{60}$ H. A. Tanaka, ${ }^{60}$ E. W. Varnes, ${ }^{60}$ F. Bellini, ${ }^{61}$ G. Cavoto, ${ }^{60,61}$ R. Faccini, ${ }^{15,61}$ F. Ferrarotto, ${ }^{61}$ F. Ferroni, ${ }^{61}$ M. Gaspero, ${ }^{61}$ M. A. Mazzoni, ${ }^{61}$ S. Morganti, ${ }^{61}$ M. Pierini, ${ }^{61}$ G. Piredda, ${ }^{61}$ F. Safai Tehrani, ${ }^{61}$ C. Voena, ${ }^{61}$ S. Christ, ${ }^{62}$ G. Wagner ${ }^{62}$ R. Waldi, ${ }^{62}$ T. Adye, ${ }^{63}$ N. De Groot, ${ }^{63}$ B. Franek, ${ }^{63}$ N. I. Geddes, ${ }^{63}$ G. P. Gopal, ${ }^{63}$ E. O. Olaiya, ${ }^{63}$ S. M. Xella, ${ }^{63}$ R. Aleksan, ${ }^{64}$ S. Emery, ${ }^{64}$ A. Gaidot, ${ }^{64}$ S. F. Ganzhur, ${ }^{64}$ P.-F. Giraud, ${ }^{64}$ G. Hamel de Monchenault, ${ }^{64}$ W. Kozanecki, ${ }^{64}$ M. Langer ${ }^{64}$ M. Legendre, ${ }^{64}$ G. W. London, ${ }^{64}$ B. Mayer, ${ }^{64}$ G. Schott,${ }^{64}$ G. Vasseur, ${ }^{64}$ Ch. Yeche, ${ }^{64}$ M. Zito, ${ }^{64}$ M. V. Purohit, ${ }^{65}$ A. W. Weidemann, ${ }^{65}$ F. X. Yumiceva, ${ }^{65}$ D. Aston, ${ }^{66}$ R. Bartoldus, ${ }^{66}$ N. Berger ${ }^{66}$ A. M. Boyarski, ${ }^{66}$ O. L. Buchmueller, ${ }^{66}$ M. R. Convery, ${ }^{66}$ D. P. Coupal, ${ }^{66}$ D. Dong, ${ }^{66}$ J. Dorfan, ${ }^{66}$ D. Dujmic, ${ }^{66}$ W. Dunwoodie,${ }^{66}$

R. C. Field, ${ }^{66}$ T. Glanzman, ${ }^{66}$ S. J. Gowdy, ${ }^{66}$ E. Grauges-Pous, ${ }^{66}$ T. Hadig, ${ }^{66}$ V. Halyo, ${ }^{66}$ T. Hryn'ova, ${ }^{66}$ W. R. Innes, ${ }^{66}$ C. P. Jessop, ${ }^{66}$ M. H. Kelsey, ${ }^{66}$ P. Kim,${ }^{66}$ M. L. Kocian, ${ }^{66}$ U. Langenegger, ${ }^{66}$ D. W. G. S. Leith, ${ }^{66}$ S. Luitz, ${ }^{66}$ V. Luth, ${ }^{66}$ H. L. Lynch, ${ }^{66}$ H. Marsiske, ${ }^{66}$ R. Messner, ${ }^{66}$ D. R. Muller, ${ }^{66}$ C. P. O'Grady, ${ }^{66}$ V. E. Ozcan, ${ }^{66}$

A. Perazzo, ${ }^{66}$ M. Perl, ${ }^{66}$ S. Petrak, ${ }^{66}$ B. N. Ratcliff, ${ }^{66}$ S. H. Robertson, ${ }^{66}$ A. Roodman, ${ }^{66}$ A. A. Salnikov, ${ }^{66}$ R. H. Schindler, ${ }^{66}$ J. Schwiening, ${ }^{66}$ G. Simi, ${ }^{66}$ A. Snyder, ${ }^{66}$ A. Soha, ${ }^{66}$ J. Stelzer, ${ }^{66}$ D. Su, ${ }^{66}$ M. K. Sullivan, ${ }^{66}$ J. Va'vra, ${ }^{66}$ S. R. Wagner, ${ }^{66}$ M. Weaver, ${ }^{66}$ A. J. R. Weinstein, ${ }^{66}$ W. J. Wisniewski, ${ }^{66}$ D. H. Wright, ${ }^{66}$ C. C. Young, ${ }^{66}$ P. R. Burchat, ${ }^{67}$ A. J. Edwards, ${ }^{67}$ T. I. Meyer, ${ }^{67}$ B. A. Petersen, ${ }^{67}$ C. Roat, ${ }^{67}$ S. Ahmed, ${ }^{68}$ M. S. Alam, ${ }^{68}$ J. A. Ernst, ${ }^{68}$ M. Saleem, ${ }^{68}$ F. R. Wappler, ${ }^{68}$ W. Bugg, ${ }^{69}$ M. Krishnamurthy, ${ }^{69}$ S. M. Spanier, ${ }^{69}$ R. Eckmann, ${ }^{70}$ H. Kim, ${ }^{70}$ J. L. Ritchie, ${ }^{70}$ R. F. Schwitters, ${ }^{70}$ J. M. Izen, ${ }^{71}$ I. Kitayama, ${ }^{71}$ X. C. Lou, ${ }^{71}$ S. Ye, ${ }^{71}$ F. Bianchi, ${ }^{72}$ M. Bona,${ }^{72}$ F. Gallo, ${ }^{72}$ D. Gamba, ${ }^{72}$ C. Borean, ${ }^{73}$ L. Bosisio, ${ }^{73}$ G. Della Ricca, ${ }^{73}$ S. Dittongo, ${ }^{73}$ S. Grancagnolo, ${ }^{73}$ L. Lanceri, ${ }^{73}$ P. Poropat, ${ }^{73,}{ }^{\S}$ L. Vitale, ${ }^{73}$ G. Vuagnin, ${ }^{73}$ R. S. Panvini, ${ }^{74}$ Sw. Banerjee, ${ }^{75}$ C. M. Brown, ${ }^{75}$ D. Fortin, ${ }^{75}$ P. D. Jackson, ${ }^{75}$ R. Kowalewski, ${ }^{75}$ J. M. Roney, ${ }^{75}$ H. R. Band, ${ }^{76}$ S. Dasu, ${ }^{76}$ M. Datta, ${ }^{76}$ A. M. Eichenbaum, ${ }^{76}$ J. R. Johnson, ${ }^{76}$ P. E. Kutter, ${ }^{76}$ H. Li, ${ }^{76}$ R. Liu, ${ }^{76}$ F. Di Lodovico, ${ }^{76}$ A. Mihalyi, ${ }^{76}$ A. K. Mohapatra, ${ }^{76}$ Y. Pan, ${ }^{76}$ R. Prepost ${ }^{76}$ S. J. Sekula, ${ }^{76}$ J. H. von Wimmersperg-Toeller, ${ }^{76}$ J. Wu, ${ }^{76}$ S. L. Wu, ${ }^{76}$ Z. Yu, ${ }^{76}$ and H. Neal ${ }^{77}$

(The BABAR Collaboration)

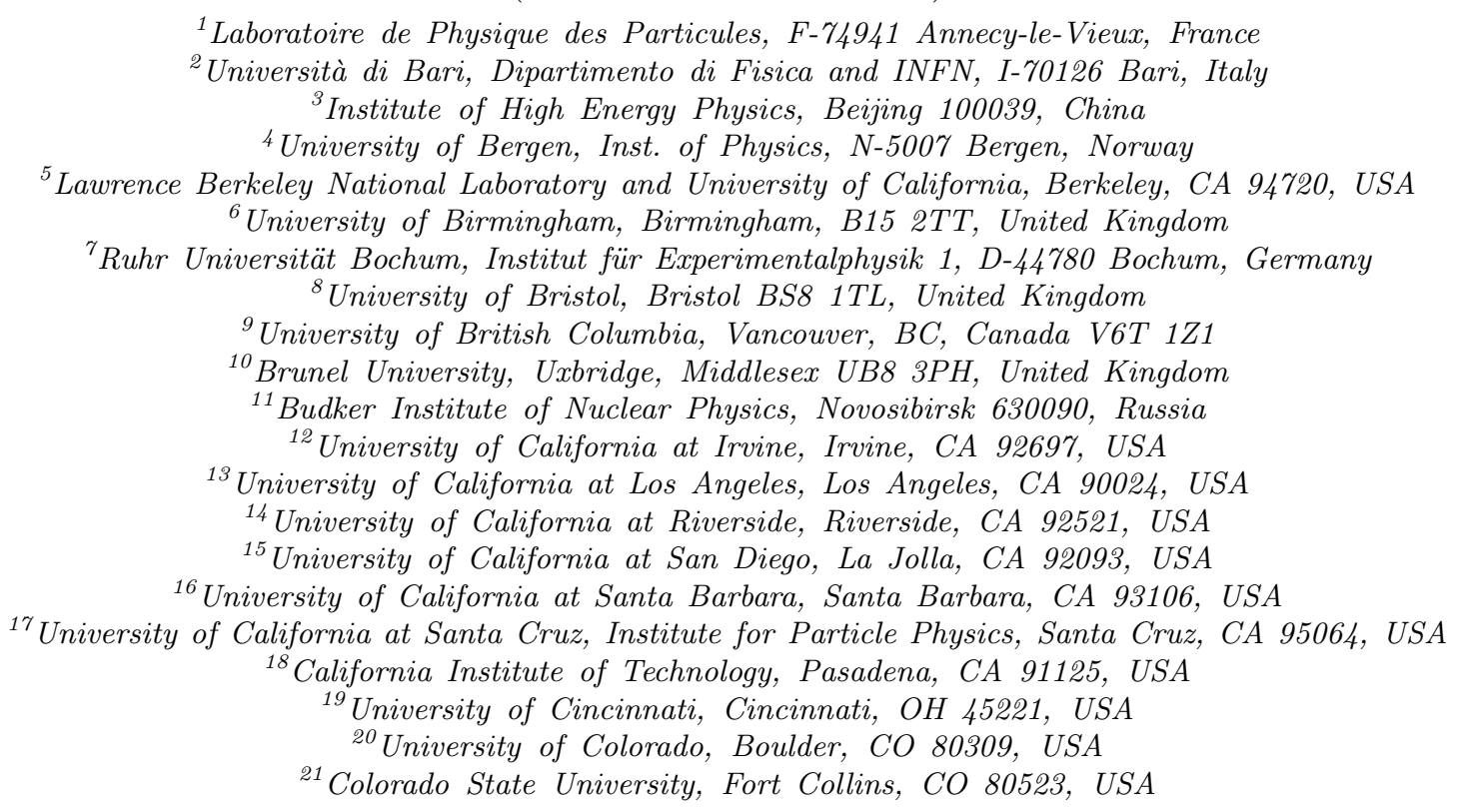


${ }^{22}$ Technische Universität Dresden, Institut für Kern- und Teilchenphysik, D-01062 Dresden, Germany

${ }^{23}$ Ecole Polytechnique, LLR, F-91128 Palaiseau, France

${ }^{24}$ University of Edinburgh, Edinburgh EH9 3JZ, United Kingdom

${ }^{25}$ Università di Ferrara, Dipartimento di Fisica and INFN, I-44100 Ferrara, Italy

${ }^{26}$ Florida A\&M University, Tallahassee, FL 32307, USA

${ }^{27}$ Laboratori Nazionali di Frascati dell'INFN, I-00044 Frascati, Italy

${ }^{28}$ Università di Genova, Dipartimento di Fisica and INFN, I-16146 Genova, Italy

${ }^{29}$ Harvard University, Cambridge, MA 02138, USA

${ }^{30}$ Imperial College London, London, SW7 $2 B W$, United Kingdom

${ }^{31}$ University of Iowa, Iowa City, IA 52242, USA

${ }^{32}$ Iowa State University, Ames, IA 50011-3160, USA

${ }^{33}$ Laboratoire de l'Accélérateur Linéaire, F-91898 Orsay, France

${ }^{34}$ Lawrence Livermore National Laboratory, Livermore, CA 94550, USA

${ }^{35}$ University of Liverpool, Liverpool L69 3BX, United Kingdom

${ }^{36}$ Queen Mary, University of London, E1 4NS, United Kingdom

${ }^{37}$ University of London, Royal Holloway and Bedford New College, Egham, Surrey TW20 0EX, United Kingdom

${ }^{38}$ University of Louisville, Louisville, KY 40292, USA

${ }^{39}$ University of Manchester, Manchester M13 9PL, United Kingdom

${ }^{40}$ University of Maryland, College Park, MD 20742, USA

${ }^{41}$ University of Massachusetts, Amherst, MA 01003, USA

${ }^{42}$ Massachusetts Institute of Technology, Laboratory for Nuclear Science, Cambridge, MA 02139, USA

${ }^{43}$ McGill University, Montréal, QC, Canada H3A 2T8

${ }^{4}$ Università di Milano, Dipartimento di Fisica and INFN, I-20133 Milano, Italy

${ }^{45}$ University of Mississippi, University, MS 38677, USA

${ }^{46}$ Université de Montréal, Laboratoire René J. A. Lévesque, Montréal, QC, Canada H3C 3J7

${ }^{47}$ Mount Holyoke College, South Hadley, MA 01075, USA

${ }^{48}$ Università di Napoli Federico II, Dipartimento di Scienze Fisiche and INFN, I-80126, Napoli, Italy

${ }^{49}$ NIKHEF, National Institute for Nuclear Physics and High Energy Physics, NL-1009 DB Amsterdam, The Netherlands

${ }^{50}$ University of Notre Dame, Notre Dame, IN 46556, USA

${ }^{51}$ Oak Ridge National Laboratory, Oak Ridge, TN 37831, USA

${ }^{52}$ Ohio State University, Columbus, OH 43210, USA

${ }^{53}$ University of Oregon, Eugene, OR 97403, USA

${ }^{54}$ Università di Padova, Dipartimento di Fisica and INFN, I-35131 Padova, Italy

${ }^{55}$ Universités Paris VI et VII, Lab de Physique Nucléaire H. E., F-75252 Paris, France

${ }^{56}$ Università di Pavia, Dipartimento di Elettronica and INFN, I-27100 Pavia, Italy

${ }^{57}$ University of Pennsylvania, Philadelphia, PA 19104, USA

${ }^{58}$ Università di Pisa, Dipartimento di Fisica, Scuola Normale Superiore and INFN, I-56127 Pisa, Italy

${ }^{59}$ Prairie View A\&M University, Prairie View, TX 77446, USA

${ }^{60}$ Princeton University, Princeton, NJ 08544, USA

${ }^{61}$ Università di Roma La Sapienza, Dipartimento di Fisica and INFN, I-00185 Roma, Italy

${ }^{62}$ Universität Rostock, D-18051 Rostock, Germany

${ }^{63}$ Rutherford Appleton Laboratory, Chilton, Didcot, Oxon, OX11 0QX, United Kingdom

${ }^{64}$ DSM/Dapnia, CEA/Saclay, F-91191 Gif-sur-Yvette, France

${ }^{65}$ University of South Carolina, Columbia, SC 29208, USA

${ }^{66}$ Stanford Linear Accelerator Center, Stanford, CA 94309, USA

${ }^{67}$ Stanford University, Stanford, CA 94305-4060, USA

${ }^{68}$ State Univ. of New York, Albany, NY 12222, USA

${ }^{69}$ University of Tennessee, Knoxville, TN 37996, USA

${ }^{70}$ University of Texas at Austin, Austin, TX 78712, USA

${ }^{71}$ University of Texas at Dallas, Richardson, TX 75083, USA

${ }^{72}$ Università di Torino, Dipartimento di Fisica Sperimentale and INFN, I-10125 Torino, Italy

${ }^{73}$ Università di Trieste, Dipartimento di Fisica and INFN, I-34127 Trieste, Italy

${ }^{74}$ Vanderbilt University, Nashville, TN 37235, USA

${ }^{75}$ University of Victoria, Victoria, BC, Canada V8W $3 P 6$

${ }^{76}$ University of Wisconsin, Madison, WI 53706, USA

${ }^{77}$ Yale University, New Haven, CT 06511, USA

(Dated: August 22, 2003)

We present a study of the decay $B^{-} \rightarrow D^{* 0} K^{*-}$ based on a sample of 86 million $\Upsilon(4 S) \rightarrow B \bar{B}$ decays collected with the BABAR detector at the PEP-II asymmetric-energy $B$ Factory at SLAC. We measure the branching fraction $\mathcal{B}\left(B^{-} \rightarrow D^{* 0} K^{*-}\right)=(8.3 \pm 1.1$ (stat) \pm 1.0 (syst) $) \times 10^{-4}$, and the fraction of longitudinal polarization in this decay to be $\Gamma_{L} / \Gamma=0.86 \pm 0.06$ (stat) \pm 0.03 (syst).

PACS numbers: $13.25 \mathrm{Hw} 14.40 . \mathrm{Nd}$ 
Following the discovery of $\mathrm{CP}$ violation in $B$-meson decays and the measurement of the angle $\beta$ of the unitarity triangle [1], focus has turned towards the measurements of the angles $\alpha$ and $\gamma$. A precise determination of $\gamma$ requires larger samples of $B$ decays than are currently available, and is likely to be based on information from several decay modes. Decays of the type $B \rightarrow D^{(*)} K^{(*)}$ are expected to play a leading role in this program [2]; among these modes, those with a $K^{*}$ have distinct advantages in some of the proposed methods [3]. Decay modes into two vector mesons present unique opportunities due to interference between helicity amplitudes. It has been suggested that angular analysis of $B^{-} \rightarrow \bar{D}^{* 0} K^{*-}$ can yield information on $\gamma$ without external assumptions [4]. More generally, such a study would be sensitive to T-violating asymmetries that probe physics beyond the Standard Model [5].

The previously available information on $B^{-} \rightarrow$ $D^{* 0} K^{*-}$ is based on a sample of 15 events [6]. Here we report on an improved measurement of the branching fraction for $B^{-} \rightarrow D^{* 0} K^{*-}$, and on the first measurement of the polarization in this decay.

Results are based on 86 million $\Upsilon(4 S) \rightarrow B \bar{B}$ decays, corresponding to an integrated luminosity of $79 \mathrm{fb}^{-1}$, collected between 1999 and 2002 with the BABAR detector at the PEP-II $B$ Factory at SLAC [7]. An additional $9.4 \mathrm{fb}^{-1}$ sample of off-resonance data, recorded at $e^{+} e^{-}$ center-of-mass (CM) energy $40 \mathrm{MeV}$ below the $\Upsilon(4 S)$ mass, is used to study "continuum" events, $e^{+} e^{-} \rightarrow q \bar{q}$ $(q=u, d, s$, or $c)$.

The BABAR detector is described elsewhere [8]. Only detector components relevant for this analysis are summarized here. Trajectories of charged particles are measured in a five-layer silicon vertex tracker (SVT) and a 40-layer drift chamber (DCH) in a $1.5-\mathrm{T}$ magnetic field. Charged particles are identified as pions or kaons using information from a detector of internally reflected Cherenkov light, as well as measurements of energy loss in the SVT and the DCH. Photons are detected in a $\mathrm{CsI}(\mathrm{Tl})$ calorimeter.

We reconstruct $B^{-} \rightarrow D^{* 0} K^{*-}$ in the following modes: $D^{* 0} \rightarrow D^{0} \pi^{0}$ and $D^{0} \gamma ; D^{0} \rightarrow K^{-} \pi^{+}, K^{-} \pi^{+} \pi^{0}$, and $K^{-} \pi^{+} \pi^{+} \pi^{-} ; K^{*-} \rightarrow K_{S} \pi^{-} ; K_{S} \rightarrow \pi^{+} \pi^{-} ; \pi^{0} \rightarrow \gamma \gamma$ (charged conjugate decay modes are implied throughout this Letter). The optimization of the event selection was based on studies of off-resonance data and simulated $B \bar{B}$ events. A key feature of the analysis is the use of a sample of $4500 \mathrm{~B}^{-} \rightarrow D^{* 0} \pi^{-}$events to validate several aspects of the simulation and the analysis procedure.

We select $K_{S}$ candidates from pairs of oppositelycharged tracks that form an invariant mass within $9 \mathrm{MeV}$ $(3 \sigma)$ of the known [9] $K_{S}$ mass. Each $K_{S}$ candidate is combined with a negatively charged track, assumed to be a $\pi^{-}$, to form a $K^{*-}$ candidate. We retain $K^{*-}$ candidates with mass within $75 \mathrm{MeV}$ of the known $K^{*-}$ mass.
The $K_{S}$ vertex must be displaced by at least $3 \mathrm{~mm}$ from the $K^{*-}$ vertex. This requirement rejects combinatorial background and is $96 \%$ efficient for real $K_{S}$ decays.

Photon candidates are constructed from calorimeter clusters with lateral profiles consistent with photon showers. Neutral-pion candidates are formed from pairs of photon candidates with invariant mass between 115 and $150 \mathrm{MeV}$. The $\pi^{0}$ mass resolution is $6.5 \mathrm{MeV}$.

We select $D^{0}$ candidates in the three decay modes listed above. To reduce backgrounds, tracks from $D^{0} \rightarrow$ $K^{-} \pi^{+} \pi^{0}$ and $D^{0} \rightarrow K^{-} \pi^{+} \pi^{+} \pi^{-}$must have momenta above $150 \mathrm{MeV}$. The kaon candidate track must satisfy a set of kaon identification criteria that provides a rejection factor of about 30 against pions. The kaon identification efficiency averaged over all kinematically allowed momentum and polar angle is $90 \%$. For each $D^{0} \rightarrow K^{-} \pi^{+} \pi^{0}$ candidate, we calculate the square of the decay amplitude $\left(|A|^{2}\right)$ based on the kinematics of the decay products and the known properties of the Dalitz plot for this decay [10]. We retain candidates if $|A|^{2}$ is greater than $5.5 \%$ of its maximum possible value. The efficiency of this requirement is $76 \%$. Finally, the measured invariant mass of $D^{0}$ candidates must be within $2.5 \sigma$ of the $D^{0}$ mass.

We select $D^{* 0}$ candidates by combining $D^{0}$ candidates with a $\pi^{0}$ or photon candidate. The $\pi^{0}$ candidate must have momentum between 70 and $450 \mathrm{MeV}$ in the $\mathrm{CM}$ frame. The photon candidate must have energy above $100 \mathrm{MeV}$ in the laboratory frame. We reject photon candidates consistent with originating from $\pi^{0}$ decay when paired with another photon of energy above $100 \mathrm{MeV}$. We require the mass difference $\Delta m \equiv m\left(D^{* 0}\right)-m\left(D^{0}\right)$ to be between 138.7 and 145.7 (130.0 and 156.0) MeV for $D^{* 0} \rightarrow D^{0} \pi^{0}\left(D^{* 0} \rightarrow D^{0} \gamma\right)$. The $\Delta m$ resolution is 1.1 (6.4) $\mathrm{MeV}$ for the $D^{0} \pi^{0}\left(D^{0} \gamma\right)$ mode.

At each stage in the reconstruction chain, the measurement of the momentum vector of each intermediate particle is improved by refitting the momenta of its decay products with kinematic constraints. These constraints are based on the known mass of the intermediate particle and on the fact that its decay products originate from a common point in space.

Finally, we select $B^{-}$candidates by combining $D^{* 0}$ and $K^{*-}$ candidates. A $B^{-}$candidate is characterized by the energy-substituted mass $m_{\mathrm{ES}} \equiv$ $\sqrt{\left(\frac{1}{2} s+\vec{p}_{0} \cdot \vec{p}_{B}\right)^{2} / E_{0}^{2}-p_{B}^{2}}$ and energy difference $\Delta E \equiv$ $E_{B}^{*}-\frac{1}{2} \sqrt{s}$, where $E$ and $p$ are energy and momentum, the asterisk denotes the $\mathrm{CM}$ frame, the subscripts 0 and $B$ refer to the initial $\Upsilon(4 S)$ and $B$ candidate, respectively, and $s$ is the square of the CM energy. For signal events we expect $m_{\mathrm{ES}}=M_{B}$ within the experimental resolution of about $3 \mathrm{MeV}$, where $M_{B}$ is the known $B^{-}$mass.

We require $|\Delta E| \leq 40 \mathrm{MeV}$ for $B^{-}$candidates with a $D^{0} \rightarrow K^{-} \pi^{+} \pi^{0}$, and $|\Delta E| \leq 27.5 \mathrm{MeV}$ for all other modes. The $\Delta E$ resolution is approximately $19 \mathrm{MeV}$ in the $K^{-} \pi^{+} \pi^{0}$ mode and $10 \mathrm{MeV}$ in the other modes. 
To reduce continuum backgrounds, we make use of the ratio of the second to zeroth order Fox-Wolfram [11] moments $\left(R_{2}<0.4\right)$, and the angle $\theta_{T}^{*}$ between the thrust axes of the $B^{-}$candidate and the remaining charged tracks and neutral clusters in the event $\left(\left|\cos \theta_{T}^{*}\right|<0.85\right)$. We also make requirements on the polar angle $\theta_{B}^{*}$ of the $B^{-}$candidate $\left(\left|\cos \theta_{B}^{*}\right|<0.9\right)$, and the energy flow in the rest of the event. We construct a Fisher discriminant $\mathcal{F}$ based on the energy flow in nine concentric cones around the direction of the $B^{-}$candidate [12]. We require $\mathcal{F}<0.40(0.28)$ for $B^{-}$candidates with a $D^{* 0} \rightarrow D^{0} \pi^{0}\left(D^{0} \gamma\right)$. The energy flow, $\theta_{T}^{*}$, and $\theta_{B}^{*}$ are all calculated in the CM frame. These requirements remove about $80 \%$ of the continuum backgrounds and are $79 \%$ (74\%) efficient for signal in the $D^{0} \pi^{0}\left(D^{0} \gamma\right)$ mode.

In $16 \%$ of the events there is more than one $B^{-}$candidate. In these events we retain the best candidate based on a $\chi^{2}$ algorithm that uses the measured values, known values, and resolutions of the $D^{0}$ mass and the mass difference $\Delta m$.

We extract the yield of $B^{-} \rightarrow D^{* 0} K^{*-}$ events from a binned maximum-likelihood fit to the $m_{\mathrm{ES}}$ distribution of $B^{-}$candidates. The signal distribution is parametrized as a Gaussian and the combinatorial background as a threshold function [13]. The parameters of the Gaussian are determined from the $m_{E S}$ distribution of the $B^{-} \rightarrow D^{* 0} \pi^{-}$sample. The total signal yield is $121 \pm 15$ events. The third column of Table I lists the yields for the individual $D^{* 0} / D^{0}$ modes. Figure 1 shows the $m_{E S}$ distribution of $B^{-}$candidates overlaid with the fit model.

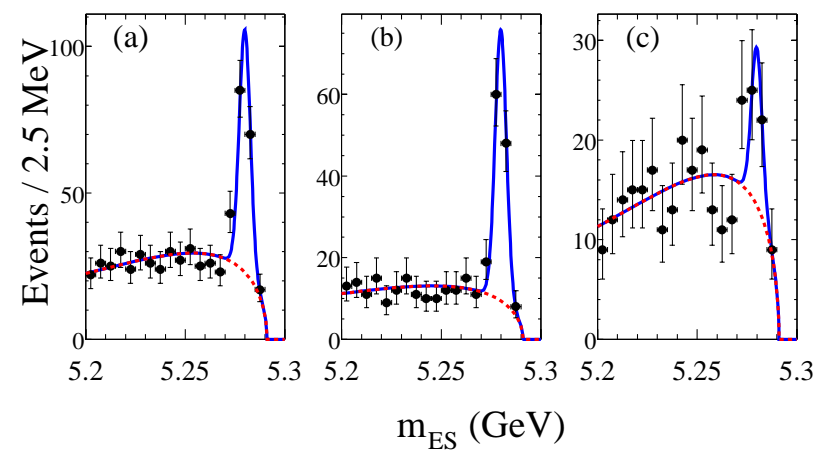

FIG. 1: Distributions of $m_{\mathrm{ES}}$ for $B^{-} \rightarrow D^{* 0} K^{*-}$ : (a) all modes; (b) $D^{* 0} \rightarrow D^{0} \pi^{0}$ modes; (c) $D^{* 0} \rightarrow D^{0} \gamma$ modes.

The yield from the $m_{E S}$ fit includes contributions from "peaking backgrounds", which are backgrounds with $m_{E S}$ near $M_{B}$. These backgrounds arise from $B$ decay modes closely related to the signal mode; e.g., $\bar{B}^{0} \rightarrow D^{*+} K^{*-}$. From a Monte Carlo simulation we estimate that they contribute $6.8 \pm 3.4$ events to the signal yield, where the uncertainty reflects the limited knowledge of the branching fractions for these modes.

The branching fraction $\mathcal{B}\left(B^{-} \rightarrow D^{* 0} K^{*-}\right)$ is calcu- lated from

$$
\mathcal{B}=\frac{N_{m_{E S}}-N_{p k}}{N_{B \bar{B}} \cdot \mathcal{B}_{K^{*-}} \cdot \mathcal{B}_{K_{S}} \cdot \sum_{i}\left(\epsilon_{M C}^{i} \cdot \mathcal{B}_{D^{* 0}}^{i} \cdot \mathcal{B}_{D^{0}}^{i}\right)},
$$

where $N_{m_{E S}}$ is the event yield from the $m_{E S}$ fit, $N_{p k}$ is the peaking background, $N_{B \bar{B}}=(85.8 \pm 0.9) \times 10^{6}$ is the number of $B \bar{B}$ pairs in the data sample, $\mathcal{B}_{K^{*-}}$ and $\mathcal{B}_{K_{S}}$ are the branching fractions for $K^{*-} \rightarrow K_{S} \pi^{-}$ and $K_{S} \rightarrow \pi^{+} \pi^{-}, i$ is an index that runs over the six $D^{* 0} / D^{0}$ modes considered in this analysis, $\epsilon_{M C}^{i}$ is the event selection efficiency, and $\mathcal{B}_{D^{* 0}}^{i}\left(\mathcal{B}_{D^{0}}^{i}\right)$ is the $D^{* 0}\left(D^{0}\right)$ branching fraction for the $i$-th mode. This calculation assumes $\mathcal{B}\left(\Upsilon(4 S) \rightarrow B^{+} B^{-}\right)=\mathcal{B}\left(\Upsilon(4 S) \rightarrow B^{0} \bar{B}^{0}\right)$. The Monte Carlo efficiency determination uses the value of the polarization reported in this Letter.

The inputs to the branching fraction calculation are summarized in Table I. Combining the six $D^{* 0} / D^{0}$ modes, we measure a branching fraction

$\mathcal{B}\left(B^{-} \rightarrow D^{* 0} K^{*-}\right)=(8.3 \pm 1.1($ stat $) \pm 1.0($ syst $)) \times 10^{-4}$.

The reconstruction efficiencies for photons and charged tracks are understood at the level of $2.5 \%$ per photon and $0.8 \%$ per track, based on studies of a variety of control samples. These are the dominant systematic uncertainties in the determination of $\mathcal{B}$. The efficiencies of many of the analysis requirements are measured in the large $B^{-} \rightarrow D^{* 0} \pi^{-}$control sample. The uncertainties on $\mathcal{B}$ are listed in Table II.

We also compute the branching fraction $\mathcal{B}\left(B^{-} \rightarrow\right.$ $D^{* 0} K^{*-}$ ) using only events from the individual $D^{* 0} / D^{0}$ modes (see Table I). The branching fractions measured in the $D^{* 0} \rightarrow D^{0} \gamma$ modes are somewhat lower than those measured in the $D^{* 0} \rightarrow D^{0} \pi^{0}$ modes. However, in the $B^{-} \rightarrow D^{* 0} \pi^{-}$sample, where the $D^{* 0}$ is reconstructed with identical techniques, we find agreement between data and expectations for the relative yields of events in all six modes. Thus, we ascribe the difference in the measured branching fractions between the modes listed in Table I to statistical fluctuations.

The angular distributions for the decay chains $B^{-} \rightarrow$ $D^{* 0} K^{*-}$ followed by $D^{* 0} \rightarrow D^{0} \pi^{0}$ or $D^{0} \gamma$ are expressed in terms of three amplitudes $H_{0}$ (longitudinal), $H_{+}$, and $H_{-}$(transverse), and three angles, $\theta_{D}, \theta_{K}$ and $\chi[14]$. The angle $\theta_{D}\left(\theta_{K}\right)$ is the angle of the $D^{0}\left(K_{S}\right)$ with respect to the $B^{-}$direction in the $D^{* 0}\left(K^{*-}\right)$ rest frame; $\chi$ is the angle between the decay planes of the $D^{* 0}$ and the $K^{*-}$ in the $B^{-}$rest frame. The experimental acceptance is nearly independent of $\chi$. Integrating over $\chi$, the angular distributions reduce to

$$
\begin{aligned}
\frac{d^{2} \Gamma}{d \cos \theta_{D} d \cos \theta_{K}} & \propto 4\left|H_{0}\right|^{2} \cos ^{2} \theta_{D} \cos ^{2} \theta_{K} \\
\frac{d^{2} \Gamma}{d \cos \theta_{D} d \cos \theta_{K}} & \propto\left(\left|H_{+}\right|^{2}+\left|H_{-}\right|^{2}\right) \sin ^{2} \theta_{D} \sin ^{2} \theta_{K}, \\
& +\left(\left|H_{+}\right|^{2} \sin ^{2} \theta_{D} \cos ^{2} \theta_{K}\right. \\
&
\end{aligned}
$$

for $D^{* 0} \rightarrow D^{0} \pi^{0}$ and $D^{* 0} \rightarrow D^{0} \gamma$, respectively. 
TABLE I: Summary of the elements of the branching fraction calculation. $N_{m_{E S}}$ is the yield from the $m_{E S}$ fit; $N_{p k}$ is the number of peaking background events; $\epsilon_{M C}^{i}$ is the event selection efficiency for the $i$-th mode; $\mathcal{B}^{i} \equiv \mathcal{B}_{K^{*-}} \cdot \mathcal{B}_{K_{S}} \cdot \mathcal{B}_{D^{* 0}}^{i} \cdot \mathcal{B}_{D^{0}}^{i}$ is the product of branching fractions for the $K^{*}, K_{S}, D^{*}$, and $D$ decays in the $i$-th mode.

\begin{tabular}{|l|l|c|c|c|c|c|}
\hline$D^{* 0}$ mode & $D^{0}$ mode & $N_{m_{E S}}$ & $N_{p k}$ & $\sum\left(\epsilon_{M C}^{i} \times \mathcal{B}^{i}\right)\left(\times 10^{-3}\right)$ & $\mathcal{B}\left(B^{-} \rightarrow D^{* 0} K^{*-}\right)\left(\times 10^{-4}\right)$ \\
\hline All & All & $121 \pm 15$ & $6.8 \pm 3.4$ & \multicolumn{2}{|c|}{$1.6 \pm 0.2$} & $8.3 \pm 1.1 \pm 1.0$ \\
\hline$D^{* 0} \rightarrow D^{0} \pi^{0}$ & All & $96 \pm 12$ & $4.8 \pm 2.4$ & \multicolumn{2}{|c|}{$1.0 \pm 0.1$} & $10.2 \pm 1.3 \pm 1.3$ \\
$D^{* 0} \rightarrow D^{0} \gamma$ & All & $24 \pm 8$ & $2.0 \pm 1.0$ & \multicolumn{2}{c|}{$0.6 \pm 0.1$} & $4.4 \pm 1.7 \pm 0.8$ \\
\hline \hline & & & & $\epsilon_{M C}^{i}$ & $\mathcal{B}^{i}$ & \\
\hline$D^{* 0} \rightarrow D^{0} \pi^{0}$ & $D^{0} \rightarrow K^{-} \pi^{+}$ & $26 \pm 5$ & $1.7 \pm 0.9$ & $(6.5 \pm 0.6) \%$ & $(0.54 \pm 0.03) \%$ & $8.0 \pm 1.8 \pm 0.9$ \\
$D^{* 0} \rightarrow D^{0} \pi^{0}$ & $D^{0} \rightarrow K^{-} \pi^{+} \pi^{0}$ & $39 \pm 8$ & $1.7 \pm 0.9$ & $(2.1 \pm 0.3) \%$ & $(1.85 \pm 0.15) \%$ & $10.9 \pm 2.4 \pm 1.7$ \\
$D^{* 0} \rightarrow D^{0} \pi^{0}$ & $D^{0} \rightarrow K^{-} \pi^{+} \pi^{+} \pi^{-}$ & $31 \pm 7$ & $1.4 \pm 0.7$ & $(2.9 \pm 0.4) \%$ & $(1.06 \pm 0.07) \%$ & $11.6 \pm 2.6 \pm 1.6$ \\
$D^{* 0} \rightarrow D^{0} \gamma$ & $D^{0} \rightarrow K^{-} \pi^{+}$ & $11 \pm 4$ & $0.1 \pm 0.1$ & $(5.7 \pm 0.5) \%$ & $(0.33 \pm 0.03) \%$ & $6.8 \pm 2.7 \pm 1.0$ \\
$D^{* 0} \rightarrow D^{0} \gamma$ & $D^{0} \rightarrow K^{-} \pi^{+} \pi^{0}$ & $11 \pm 5$ & $1.7 \pm 0.9$ & $(1.9 \pm 0.2) \%$ & $(1.14 \pm 0.12) \%$ & $5.3 \pm 2.9 \pm 1.0$ \\
$D^{* 0} \rightarrow D^{0} \gamma$ & $D^{0} \rightarrow K^{-} \pi^{+} \pi^{+} \pi^{-}$ & $0 \pm 5$ & $0.2 \pm 0.1$ & $(2.5 \pm 0.3) \%$ & $(0.65 \pm 0.07) \%$ & $-0.2 \pm 3.3 \pm 0.4$ \\
\hline
\end{tabular}

TABLE II: Uncertainties on $\mathcal{B}\left(B^{-} \rightarrow D^{* 0} K^{*-}\right)$.

\begin{tabular}{|l|c|}
\hline Source & Uncertainty \\
\hline Statistical & $13.1 \%$ \\
\hline$\pi^{0}$ and $\gamma$ efficiency & $6.0 \%$ \\
Tracking efficiency & $4.5 \%$ \\
$m_{\text {ES fitting assumptions }}$ & $3.8 \%$ \\
Event selection criteria & $3.8 \%$ \\
$D^{* 0}$ and $D^{0}$ branching fractions & $3.2 \%$ \\
Peaking background estimates & $3.0 \%$ \\
Kaon identification efficiency & $2.0 \%$ \\
$K_{S}$ efficiency & $1.9 \%$ \\
Polarization uncertainty & $1.8 \%$ \\
Monte Carlo statistics & $1.7 \%$ \\
$N_{B \bar{B}}$ & $1.1 \%$ \\
\hline Total Systematics & $11.7 \%$ \\
\hline
\end{tabular}

The longitudinal polarization fraction $\Gamma_{L} / \Gamma$, given by

$$
\frac{\Gamma_{L}}{\Gamma}=\frac{\left|H_{0}\right|^{2}}{\left|H_{0}\right|^{2}+\left|H_{+}\right|^{2}+\left|H_{-}\right|^{2}}
$$

is extracted from an unbinned maximum-likelihood fit to the $\left(\theta_{D}, \theta_{K}\right)$ distribution for events with $m_{E S}>5.27$ $\mathrm{GeV}$. The data distribution $(D)$ is fit to the sum of distributions for longitudinally $(L)$ and transversely $(T)$ polarized signal events, and combinatorial background events $(C)$ :

$D\left(\theta_{D}, \theta_{K}\right)=a \cdot L\left(\theta_{D}, \theta_{K}\right)+b \cdot T\left(\theta_{D}, \theta_{K}\right)+c \cdot C\left(\theta_{D}, \theta_{K}\right)$.

Here $c$ is the fraction of combinatorial background determined from the $m_{E S}$ yield fit, and $b=1-a-c$. Thus, $a$ is the only free parameter in the fit.

The distributions of $L$ and $T$ are obtained from simulations of transverse and longitudinal decays, including detector acceptance effects. The distribution of $C$ is estimated from data candidates in a sideband of $m_{\mathrm{ES}}$ $\left(5.20<m_{\mathrm{ES}}<5.27 \mathrm{GeV}\right)$. We exclude from the fit $\left(\theta_{D}, \theta_{K}\right)$ regions where the efficiency changes rapidly: $\cos \theta_{K}<-0.9$ and, in the $D^{0} \gamma$ mode, $\cos \theta_{D}>0.85$.

We find longitudinal polarization fractions $\Gamma_{L} / \Gamma=$ $0.87 \pm 0.07$ (stat) \pm 0.03 (syst) and $0.80 \pm 0.14$ (stat) \pm
0.04 (syst) from fits to the $D^{* 0} \rightarrow D^{0} \pi^{0}$ and $D^{* 0} \rightarrow D^{0} \gamma$ samples, respectively. Figure 2 shows projections of the $\left(\theta_{D}, \theta_{K}\right)$ distributions for the event sample.

Combining the results from the two $D^{* 0}$ modes, we find $\Gamma_{L} / \Gamma=0.86 \pm 0.06$ (stat) \pm 0.03 (syst). The systematic uncertainty reflects the accuracy of the simulation $( \pm 0.017)$, the uncertainty on the fraction $c( \pm 0.017)$, the finite statistics of the simulation $( \pm 0.010)$, the uncertainties related to the fit assumptions $( \pm 0.010)$, and the uncertainty due to the assumption that the acceptance is independent of $\chi( \pm 0.004)$. As a consistency check, we fit the $\theta_{D}$ distribution in the $B^{-} \rightarrow D^{* 0} \pi^{-}$sample. We find $\Gamma_{L} / \Gamma=1.00 \pm 0.01$, in agreement with the expectation $\Gamma_{L} / \Gamma=1$ from angular momentum conservation.
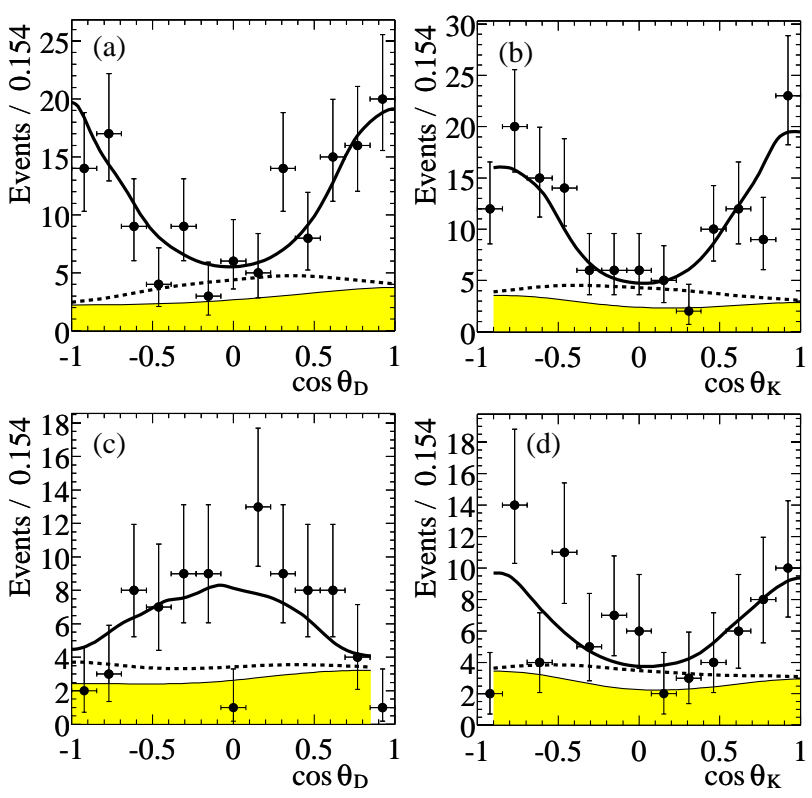

FIG. 2: Distributions of (a) $\cos \theta_{D}$ and (b) $\cos \theta_{K}$ for $D^{* 0} \rightarrow$ $D^{0} \pi^{0}$. Distributions of (c) $\cos \theta_{D}$ and (d) $\cos \theta_{K}$ for $D^{* 0} \rightarrow$ $D^{0} \gamma$. The solid line represents the full fit model, the dashed line represents the transverse component, and the shaded region represents the combinatorial background component. 
In summary, we have measured the branching fraction for $B^{-} \rightarrow D^{* 0} K^{*-}$ to be $\mathcal{B}=(8.3 \pm 1.1$ (stat) \pm 1.0 (syst) $) \times 10^{-4}$. Our measurement is a factor of 2.5 more precise than the previous result. It is in agreement with predictions based on the measured $B^{-} \rightarrow D^{* 0} \rho^{-}$ branching fraction [15], and the value of the Cabibbo angle. We have also measured the longitudinal polarization fraction in this decay to be $\Gamma_{L} / \Gamma=0.86 \pm 0.06$ (stat) \pm 0.03 (syst). This last result is consistent with expectations [16] based on factorization, Heavy Quark Effective Theory, and the measurement of semileptonic $B$ decay form factors, assuming that the external spectator amplitude $\left(b \rightarrow c W^{*-} ; W^{*-} \rightarrow K^{*-}\right)$ dominates in $B^{-} \rightarrow D^{* 0} K^{*-}$.

We are grateful for the excellent luminosity and machine conditions provided by our PEP-II colleagues, and for the substantial dedicated effort from the computing organizations that support BABAR. The collaborating institutions wish to thank SLAC for its support and kind hospitality. This work is supported by DOE and NSF (USA), NSERC (Canada), IHEP (China), CEA and CNRS-IN2P3 (France), BMBF and DFG (Germany), INFN (Italy), FOM (The Netherlands), NFR (Norway), MIST (Russia), and PPARC (United Kingdom). Individuals have received support from the A. P. Sloan Foundation, Research Corporation, and Alexander von Humboldt Foundation.

* Also with Università di Perugia, Perugia, Italy

$\dagger$ Also with Università della Basilicata, Potenza, Italy

* Also with IFIC, Instituto de Física Corpuscular, CSICUniversidad de Valencia, Valencia, Spain
$\S$ Deceased

[1] BABAR Collaboration, B. Aubert et al., Phys. Rev. Lett. 89, 201802 (2002); Belle Collaboration, K. Abe et al., Phys. Rev. D66 071102 (2002).

[2] M. Gronau and D. Wyler, Phys. Lett. B265, 172 (1991); I. Dunietz, Phys. Lett. B270, 75 (1991); D. Atwood, G. Eilam, M. Gronau, and A. Soni, Phys. Lett. B341, 372 (1995); D. Atwood, I. Dunietz, and A. Soni, Phys. Rev. Lett. 78, 3257 (1997).

[3] J.H. Jang and P. Ko, Phys. Rev. D58, 111302 (1998); M. Gronau and J.L. Rosner. Phys. Lett. B439, 171 (1998).

[4] N. Sinha and R. Sinha, Phys. Rev. Lett. 80, 3706 (1998).

[5] D. London, N. Sinha, and R. Sinha, hep-ph/0304230.

[6] CLEO Collaboration, R. Mahapatra et al., Phys. Rev. Lett. 88, 101803 (2002).

[7] PEP-II Conceptual Design Report, SLAC-0418 (1993).

[8] BABAR Collaboration, B. Aubert et al., Nucl. Instr. and Methods A479, 1 (2002).

[9] We take particle masses from Particle Data Group, K. Hagiwara et al., Phys. Rev. D66, 010001 (2002).

[10] CLEO collaboration, S. Kopp et al. Phys. Rev. D 63, 092001 (2001)

[11] G.C. Fox and S. Wolfram, Phys. Rev. Lett. 41, 1581 (1978).

[12] CLEO Collaboration, D.M. Asner et al., Phys. Rev. D53, 1039 (1996).

[13] The function is $f\left(m_{E S}\right) \propto m_{E S} \sqrt{1-x^{2}} \exp \left[-\zeta\left(1-x^{2}\right)\right]$, where $\zeta$ is a fit parameter, $x=m_{E S} / m_{0}$, and $m_{0}$ is onehalf the average CM energy; ARGUS Collaboration, H. Albrecht et al., Phys. Lett. B192, 245 (1987).

[14] See for example G. Kramer and W.F. Palmer, Phys. Rev. D45, 193 (1992).

[15] CLEO Collaboration, S. E. Csorna et al., Phys. Rev. D67, 112002 (2003).

[16] J. Korner and G. Goldstein, Phys. Lett. B89, 105 (1979); M. Neubert and B. Stech, in Heavy Flavors, 2nd Edition, edited by A.J. Buras and M. Lindner, World Scientific, Singapore, 1997. 